cerebral disease, attended with insensibility, or with perversion of the intellectual powers; while, on the other hand, I cannot too highly extol the capious abstraction of blood in appropriate cases. The state of the pulse I believe to be an unfailing criterion as to the necessity of blood-letting, which, when performed judiciously, and in time, has often in my own experience, and no doubt in that of many others, succeeded in averting the most formidable and fatal attacks of Cerebral Diserse.

8, Torrngton Square, March 1850.

\title{
ON THE PROGNOSIS AND TREATMENT OF ORGANIC DISEASES OF THE HEART.
}

By CHARLES J. B. WILLIAMS, M.D., F.R.S.

[Conchuded from last Number, p. 320.]

We find, then, that Strocturai, Diseases of the Heart are by no means universally dangerous or fatal in their tendency; but that they are so in proportion as the circulation becomes seriously impaired, either through the extent of the cardiac lesion, or through the additional disordering influence of an ill-proportioned quantity, or a depraved quality, of the blood. It is therefore obvious, that it should be the object of the Treatment, in maladies of this nature, to counteract or correct, so far as is possible, those influences which may give to each case a formidable character, and to place the patient under circumstances that shall favour and aid the damaged organ in carrying on the work of the circulation. Nor are these objects so unattainable in the plurality of cases, as might be expected from the vital value of the organ implicated. I know of few instances of structural disease of an important viscus that admit of more mitigation, under judicious treatment, than many Organic Affections of the Heart; and the examples, to which I have already alluded, of these affections enduring for a very long period, gradually increasing to a prodigious extent without destroying life, and sometimes without greatly sacrificing the comfort of the patient, give further proofs that the powers of adaptation and endurance, in this most vital of all organs, are far greater than is commonly imagined.

It may readily be inferred from my former remarks on Organic Diseases of the Heart, that the benefit which the subjects of them derive from medical treatment, depends on neither the activity or energy of the practice employed, nor on any uniformity of plan. In this, as in most intelligible cases of disease, the best practice is that rationally adapted to varying elements of disorder, with a view to restore that balance of functions which is most favourable to the easy action of the defective organ.

I have alieady noticed the prevailing tendency among many practitioners to treat Organic Diseases of the Heart by sedatives, and other lowering or reducing measures; but, according to my experience, such treatment is applicable only to periods of excitement or exacerbation, and the plan most generally useful, is one calculated to sustain the 
pawens of the circulation. There is much that is fictitious and partial in the inordinate action and palpitation of a diseased heart. The violent knock against the ribs, and the strong throbbing in the large arteries, are evidences of a force that is misdirected and ineffectual rather than excessive ; for, at the same time, the pulse of distant arteries is scarcely to be felt, the extremities are cold, and the capillary circulation generally feeble. Hence, although sedatives may be beneficial as temporary expedients, measures of a sustaining and strengthening nature are more permanently useful. Organic lesions of the heart, in most instances, impair its propulsive power; and it is quite reasonable to expect most good from the means which shall have a contrary influence, by augmenting the steady strength of the organ, by improving the quality of the blood, and by promoting its equal distribution by the removal of obstructions and congestions. It is equally apparent, on the other hand, that these objects are not to be fulfilled by mere stimulants and tonics. The fact is quite familiar to practitioners, that in many instances of Heart Disease, the common spirituous and ethereal stimulants, and the stronger tonics, such as iron and quinine, aggravate the symptoms, and induce attacks of palpitation, oppression, and lividity. Yet this, as well as the opposite class of remedies, is not only borne, but proves most salutary at a proper season and under fitting circumstances. In order to know these seasons and circumstances, it is necessary to revert to the sketch of the pathology of Diseases of the Heart, already given in relation to Prognosis, and to consider it again in its application to practice.

We may conveniently consider the subject under the following heads of Indications of Treatment.

1. To regulate and promote the moderate and efficient Action of the Heart itself.

2. To improve the Quality, and regulate the Quantity and Distribution of Blood throughout the body.

3. To prevent or counteract the Lesions of Function and Structure which supervene in other organs, in the progress of Heart-Disease.

1. The treatment addressed to the Condition of the Heart itself will vary considerably, not only in different cases, but in the same case at different times. At early periods of organic disease, whether of the valves, investments, or muscular structures, there is commonly a tendency to excitement, manifested by increased action, palpitation, and sometimes pain or some kind of uneasiness in the cardiac region. In inflammatory and plethoric suhjects, this excitement may either amount to inflammation, or assume some of those forms of excessive and vitiated local nutrition, which tend to further disorganization of the organ. Under such circumstances, there can be no doubt of the propriety of antiphlogistic measures, proportioned to the severity of the symptoms. In the absence of marked plethora or decided pyrexia, local depletion or counter-irritation will generally suffice, especially if aided by the means afterwards to be noticed for reducing the stimulating property of the blood.

The instances in which general blood-letting is called for in Organic Diseases of the Heart, are, according to my experience, rare. I do not deny that it will often, for the time, relieve strong action of the heart and vascular excitement more promptly than any other means; but it 
generally leaves afterwards a weakness and irritability of the organ, which unfits it for the regular work of the circulation, and which, therefore, so tends to the recurrence of congestion, oppression, and excitement, as to call for a repetition of the blood-letting. Thus the blood becomes impoverished, and the constitutional powers lowered; and not only is there added the complication of anæmia, which I before noticed as a common result of such treatment, but the power of the excreting organs fails also, and cachæmia, dropsy, and degenerations, may ensue. As in other cases of defective organization, it is a great object to sustain the constitutional powers; and we must not lose sight of this in our endeavours to reduce local inflammation and temporary excitement.

Cupping, leeching, and blistering in the region of the heart, aided by mild mercurials, antimonials, sedatives, and salines, with moderate diet and rest, will be found safer measures than venesection, and are generally adequate to reduce the excitement of the heart, even in inflammatory and plethoric subjects. Dry cupping extensively used, ${ }^{3}$ and countercongestion by hæmostatic ligatures on one or more limbs, (as for venesection), are also available means for relieving the heart from the weight and stimulus of the blood, during periods of temporary sthenic excitement.

But in many examples, particularly in persons of nervous temperament, in females, and in connexion with dyspeptic and other sympathetic disorders, the excitement of the heart is much more nervous than vascular. The suddenness and variability of the paroxysms of palpitation or painful action; their disproportion to other symptoms; the prevalence of other signs of nervous excitement or disturbance, such as spinal or superficial cutaneous tenderness, various spasms, globus hystericus, copious diuresis, etc; , will generally guide to the distinction of this kind of inordinate action : and if the subject be not positively anæmic, there will generally be sufficient evidence of the absence of any real strength in the vascular system, in the general pallidity and low temperature of the surface and extremities, and the weakness of the pulses in the limbs, as compared with those of the palpitating heart. Under such circumstances, nervous sedatives are more appropriate than depletion; and local irritation or partial fulness of blood is sufficiently counteracted by derivants, evacuants, and means which increase the natural excretions. Of the class of nervous sedatives, those which have an obvious influence on the Heart are to be preferred, especially the following, which I place in the order in which I find them most effectual : hydrocyanic acid, aconite, digitalis, camphor, and henbane. The sedative action of all these is promoted by combining them with an alkaline carbonate, which fulfils another indication to be noticed hereafter. In the hysterical or otherwise more nervous kinds of palpitation, the medicines generally called antispasmodic, such as the foetid gums, valerian, bella-

1 To save the blood by $d r y$ cupping, the exhaustion of the glass should not be carried so far as to induce much ecchymosis. If blood be extravasated, it is lost as blood ; and if reabsorbed, can be nseful only through a new process of solution and easimilation. Even in the process of counter-congestion, the blood may be injured by long stagnation; as I have shown it to be spoiled and unfit for further use, afler it has been long pent up in congested organs in intermittent and adynamic fermer. Principles of Medicine, second odition, Pp. 118, 196. 
donna, stramonium, and æther, mostly in conjunction with the volatile alkali, will be found to afford more relief. It is curious to observe how, under the influence of some of these which may be considered stimulant, the heart's action sometimes becomes calmed; but it would be com. monly found in such cases, that although the organ throbs violently, it does not effectually expel its contents, and the pulse of distant arteries is extremely weak : the diffusible stimulant, by exciting a more energetic contraction, propels the oppressive load of blood, and restores the equality and quietude of the circulation. The combination of the two kinds of medicaments, the nervous sedatives with the muscular stimu. lants, is neither irrational in theory, nor inefficacious in practice; for I have frequently obtained from the united operation of hydrocyanic acid and valerian, aconite and galbanum, or digitalis and æther, marked advantage, which the agents separately failed to afford. Another expedient which, in a few instances, has proved very effectual in subduing the violent action of a palpitating heart, is a copious draught of cold water. This may operate sympathetically, by soothing the heated and irritable surface of the stomach; but $I$ am inclined to consider its operation in some measure direct on the heart itself, which is quite near enough to the stomach and æsophagus to receire the impression of cold from a large bulk of water. Small pieces of ice swallowed in succession have a similar effect.' This measure would not be expedient in persons liable to syncope; for this opposite condition may result from the sudden reduction of the palpitation by cold.

The measures hitherto recommended are chiefly of a temporary nature, to subdue or moderate excessive action of the heart, when it is first developed, or aggravated during seasons of excitement. There wre others of more permanent operation, inadequate and even unsuited for these occasions, but applicable in the intervals between the attacks, to which they in some measure may prove preventives. I allude to the agents generally called tonic, which are, much more generally than is commonly supposed, applicable to the treatment of the Organic Dise:ses of the Heart. Much caution and discretion are necessary in their selection and administration. Thus, in the more inflammatory cases, nothirg stronger than the mineral acids or weak vegetable astringents may be borne. A class somewhat less sthenic may tolerate and receive benefit from salts of zinc or silver, tannic or gallic acids, and weak chalybeates combined with salines. A third group, bordering more on the anæmic and irritable condition, with much action but no strength, bear the more decided tonics of iron and quinine; especially when guarded by some of the sedatives before noticed, such as hydrocyanic acid, aconite, etc. But for the useful and even safe administration of all tonic remedies in connexion with an injured and irritable heart, it is essential that the secretions be free; and, in order to ensure this, it is often necessary to resort to the means to be presently noticed, as teriding to maintain the purity of the blood. It is quite intelligitle that tonics, which, in a relaxed system with free secretions, fortify the muscular and vascular

1 In my experiments on animals, the power of alight applications of cold water, to moderate the turbulence and increase the steady force of the exposed heart, was, in some instances, very remarkable; but when froely used, it caused a quivering contraction, which soon suspended all movement. 
fibres by bracing them and promoting their contractile power, may further obstruct those secreting organs whose function is imperfect, and thus add to the disorder which their failure occasions.

We have next to notice the opposite dynamic condition of the Diseased Heart, that of defective action. In many instances, this is combined with irregularity of rhythm, or inequality of pulse; but the irregularity and the failure are by no means proportioned to each other: for weak hearts sometimes show no intermission in their action; and considerable irregularity may occur without any prominent signs of weakness. Still, as a general rule, it may be laid down, that irregularity of rhythm, and inequality of strength in the pulse, belong to the class rather of defective than of excessive power of the heart, especially as regards its efficiency in carrying on the circulation. More or less irregularity or inequality of pulse is commonly observed in connexion with the following organic lesions, which distinctly impair the propulsive power:-Extensive valvular disease, especially of the mitral orifice:1 dilatation with or without moderate hypertrophy; fatty degeneration and softening, connected with partial obstruction of the coronary arteries. Advanced stages of severe pericarditis, and adhesions of the pericardium, present a remarkable kind of inequality, and even intermission, in the radial pulse, whilst the heart's pulsations are quite regular; in fact, some of the latter are too weak to reach the wrist, hence the irregularity; and I have distinctly ascertained that the weak, or deficient beats, are those corresponding with inspiration, whilst the stronger pulses are those enforced by the expiratory act, which thus helps the weak heart, and augments its propulsive power. So it often happens, that the act of coughing gives a momentary strength to a weak pulse.

It must not be inferred from the preceding remarks, that irregular action is necessarily connected with organic disease. Nothing is more common, than to find the heart acting irregularly when exhausted from fatigue or other undue excitement; or when disturbed by deranged digestion, bilious disorder, or a gouty, or otherwise depraved state of the blood. These conditions are only temporary, and amenable to treatment; but if the irregularity should continue for several weeks, in spite of treatment, there is reason to suspect the existence of structural disease; and, in the absence of signs of valvular lesions and dilatation, fatty degeneration or softening may be apprehended. ${ }^{2}$

It may well be anticipated, that the chief remedies to be recommended for defective and irregular action of the heart, are of the stimulating and sustaining kind; but although this so far holds good that it may be taken as the rule, it cannot be applied without considerable qualification

1 Obstructive lesions more particularly. In my experiments on animals, I found that nothing disturbed the regularity of the heart's action so much as obstruction of the left auriculo-ventricular orifice, by pushing the walls of the auricle into ii whis the finger.

a very complete and interesting memoir on Fatty Diseases of the Heart has recently been read to the Royal Medical and Chirurgical Society, by my friend Dr. R. Quain ; and, when published, will be found to contain the most valuable information on the history and pathology of these affections. Dr. Quain has completely verified my inference, that fatty degeneration is a chemical change, like the opontaneous change of muscle into adipocere. [An abstract of this paper will be given in our Keports of Societies, which, from the unexpected length of the Original Articles in the present number, we are reluctintly obliged to defor till Jinne. Eidross.] 
and tact. The same organic lesion in the heart which forms an objection to the unguarded use of depletion and reducing means during inordinate action, is an obstacle to the free administration of stimulants and tonics during depressed or irregular action. It must not be forgotten that we have to deal with a damaged mechanism, which, in a state of weakness, may as ill bear the goad of a stimulant, or the bracing of a tonic, as, in a state of excitement, it can sustain the loss of much blood, or of that which contributes to its regular support. In syncope, or extreme weakness, with a healthy heart, we need not fear to excite its failing powers by strong stimulants, and to maintain them by the most bracing tonics; but in a diseased organ, similar forced excitement may strain, irritate, or rupture the faulty structure, before it can restore the balance of the wanting power.

It often happens, therefore, that we have to premise or combine with the use of stimulants and tonics, means to relieve the heart of some of the oppressive load which it cannot propel. A few leeches, a moderate cupping, or a blister over the heart, or, in slighter cases, derivants to the extremities and secreting organs, may accomplish this end, and enable the stimulant to operate on the contractile power of the organ with greater certainty and safety. So, too, the various aids to an embarrassed circulation, afforded by warmth to the surface and extremities, gentle friction, and appropriate changes of posture or exercise, will generally be noeded during the continued action of tonies. With due attention to these points, most cases of Organic Disease of the Heart with defective action may be bettered for a time with tonics; and if under this treatment the local lesion, being of the nature of chronic inflammation, should show any tendency to increase, counter-irritation, with the moderate internal use of mercury or iodide of potassium, may be employed to connteract it. The tonics most suitable to augment the strength of the heart have already been noticed; for the condition of habitually defective action, the strongest kinds, if borne, are to be preferred, such as iron, quinine, tannic and gallic acids. Advantage sometimes results from combining two of these that do not form an insoluble compound, as sulphates or citrates of quinine and iron or zinc, and tannate of quinine. But the selection must also much depend on the condition of the blood as well as on that of the heart, and to this subject we now proceed.

2. The regulation of the Quantity and Condition of the Blood is an object of great importance in diseases of the organ which, more than any other, is liable to be influenced by the blood. But it will not be needful to dwell long on the means by which this indication is to be fulfilled, because they are the same as are usual in diseases of the blood. Thus, in states of plethora, the excessive quantity and richness are reduced by depletion, evacuants, and low diet; but, for reasons before stated, it is needful to take care that these be not carried too far. In asthenic plethora, the purification of the blood, by increasing the secretions and promoting its more active and steady circulation, is of more avail than depletion; this subject will again claim our attention. A cause of temporary plethora, which often seriously operates in aggravating Heart Disease, is too liberal an indulgence in liquids of any kind, especially during meals. Experience often tenches patients the advantage of moderation in this respect; and as copious libations at meals 
are more the result of habit than of real want, and are frequently sub: stituted for slow and careful mastication, there is good reason to dis: courage them. If thirst should prevail, or if a freer supply of liquid is wanted to help the processes of elimination, water, or some very simple beverage, may be taken on an empty stomach, when it is more speedily distributed through the alimentary canal, and, being absorbed, promptly passes off by the kidneys and skin.

I have already adverted to the common combination of structural Disease of the Heart with anæmia or chlorosis. As before hinted, the scantiness and poverty of the blood is frequently the result of the injudicious employment of depletion, low diet, confinement, and other lowering measures, by which it has been erroneously supposed that Diseases of the Heart are to be controlled. In other instances, however, especially after rheumatism, by whatsoever means treated, the anæmic condition arises spontaneously ; and apparently proceeds from want of power in the system to renew the blood that becomes depraved and effete by the morbid changes which have taken place in it. After other acute diseases, such as inflammations and fevers, which injure the quality of the blood, the same deficiency of red particles often occurs; and if there be a lesion of the heart present, its impairing influence on the circulation is more seriously felt. The treatment usually proper for anæmia is required for these cases; and $I$ have before mentioned, that the heart symptoms are ultimately relieved, as the heart and vessels become invigorated by more and better blood : but here too, the presence of the Heart Disease renders it necessary to proceed cautiously and slowly in the administration of iron and other tonics. The first doses often aggravate the pain and palpitation of the heart; and it is therefore expedient that they should be small, and combined with hydrocyanic acid or some other sedative, and increased gradually, so soon as they are tolerated. As, in most cases of chlorosis, the secreting organs commonly require the aid of gentle aperients and diuretics, so these are particularly needful here, where any retention of excrementitious matter would tend to irritate the damaged organ, and render it intolerant of the bracing influence of iron. Under similar circumstances, the iodide of iron has appeared to answer better than other preparations, probably from its influence on the capillary circulation and secretion; or a combination of iodide of potassium with the citrate or acetate of iron, which is less liable to decomposition than the iodide of iron itself. In the anæmic state of the system with structural Disease of the Heart succeeding to rheumatism, this combination is especially salutary.

It is not necessary to dwell on further details of the treatment calculated to improve the quantity and quality of the blood in connexion with Heart Disease; but it would not be right to omit the mention of the salutary influence of pure country air, and the full effulgence of a sunny sky, on the sanguiferous system. I have known several instances, in which, with considerable organic disease, invalids have attained a degree of strength and constitutional vigour, by passing the greater part of the day sub dio, which they never could gain from any system of medication. A long sea voyage has a like salutary influence. but it would not be safe for those liable to severe sea-sickness.

Further in relation to the blood, we have to notice the means by 
which its quality is to be improved by the removal of excrementitious and other noxious matters. Of the importance of these we bave already bad so many proofs in almost every bearing of the subject, that we have been obliged to anticipate much of what belongs to this head. In every degree and kind of Organic Disease of the Heart, we find that a deficient quantity or vitiated quality of the excretions is the common precursor or concomitant of aggrarated symptoms; and from the marked manner in which remedies which augment and improve the secretions bring relief, we are constrained to infer, that the failure of this purifying process, and the consequent accumulation of noxious matters in the blood, is the common cause of the exacerbations of Heart Disease. And in truth, it will be found that most of the various disturbing influences that may be regarded as the ladentia in Heart Disease, agree in this respect, that directly or indirectly, they impair the processes of elimination, and that their permanent operation is chiefly connected with this effect. Thus over-exertion of the body, or agitation of the mind, bring on palpitations, pain, and other severe symptoms ; but rest and tranquillity will generally remove these, provided the secretions continue free. But if the secretions have become impaired, which they commonly are where the fatigue or excitement have been considerable, then no relief ensues till these functions are restored. A fit of indigestion, exposure to cold, or confinement in an impure atmosphere, may bring on a severe aggravation of the symptoms of Heart Disease. This will be transient or enduring, generally in proportion as the processes of secretion by the kidneys, bowels, and skin, are slightly or seriously deranged. In a similar manner, the onset of febrile diseases, influenza, gout, rheumatism, erysipelas, and the like, attended as they always are by impaired secretions, never fails to bring on an exacerbation of the symptoms of Organic Disease of the Heart.

This very general fact with regard to the aggravations of Heart Disease renders intelligible what is quite familiar to experienced practitioners, although they may explain it in different ways, that the best remedies under these circumstances are those which increase the secretions of the liver and intestines, the kidneys, and the skin. A few doses of mercurial aperients, with salines and other diuretics and diaphoretics, do more to relieve the symptoms than any treatment exclusively addressed to the heart; and if they do not succeed in mitigating the more distressing parts of the malady, they at least prepare the system for sedatives, antispasmodics, and tonics, which then act with safety and effect.

In cases, therefore, of exacerbation of the symptoms of Organic Disease of the Heart, sometimes in addition to, sometimes without the measures before recommended to affect the organ primarily, we commonly administer a mercurial purgative every night or every other night, until the secretions from the intestines give evidence of a free elimination of bile. We may combine with the mercurial a little squill and digitalis, with the view to excite a fuller flow of urine; and to promote the same object, we exhibit various saline solutions with an excess of alkali or of its carbonate, during the day. These are the medicines which I find most

1 The salts of soda, potash, and ammonia, with vegetable acids, or with nitric or phosphoric acid, answer the best. 
constantly useful in relieving the exacerbations of Heart Disease, through their action on the secretions; but there are many others capable of giving supplementary or succedaneous aid, or that are specially suited to particular cases. Of these I would mention colchicum, iodide of potassium, hydrochlorate of ammonia, benzoate of ammonia or potash, taraxacum, tincture of cantharides, compound spirit of horseradish, and infusions of uva ursi, pareira, and digitalis, as those which have the least frequently disappointed me in their effects. So soon as some or other of these medrcines, alone or in combination, have sucoeeded in restoring the secretion which was defective or depraved, something of a strengthening kind, such as a bitter tonic, or chalybeate, may be advantageously added, as it will counteract the weakening operation of the eliminatory medicines, and improve the powers of digestion and assimilation.

Besides improving the quality of the blood, we have, comprised under the present head, the indication to promote, regulate. or equalize its distribution. The means which best contribute to the fulfilment of this indication, are chiefly the various expedients which promote the circulation of the surface and extremities, such as gentle exercise, friction, warm clothing, and artificial warmth. In proportion as these means augment the quantity and accelerate the flow of blood in the superficial and extreme vessels, the heart will be lightened of some of its load, and internal organs will be relieved of some of their congestion. It is obvious that these measures will be more salutary in cold, than in warm seasons : in fact, in very hot weather, they may become hurtful, by withdrawing from the heart too much of its proper stimulus, and thus inducing faintness and extreme weakness. Under these circumstances, cool astringent applications to the surface, as by sprinkling with water, sponging with vinegar, and the refreshing influence of a cool breeze, with the horizontal posture and rest, will restore the circulation and help the flagging heart.

3. The last indication is to prevent or counteract the Lesions of Function and Structure, which supervene in other organs in the progress of Heart Disease. The measures which we have just recommended as suitable to improve the condition of the blood, are those also which succeed best in correcting functional disease, and preventing structural changes; but a glance at the tabular sketch before given, of the secondary effects of Diseased Heart on other organs, suggests the importance of directing the treatment especially towards any of these that give signs of suffering. It may be observed, that the first morbid condition of the organs thus secondarily affected is, for the most part, congestion, or irregular flow of blood with disordered secretion: then follow exudations or deposits, altering the structures, and more permanently impairing the functions : lastly ensue the contraction, induration, or fatty or calcareous degeneration of these deposits, still further subverting the organisation and reducing the vitality of the structures. It is not necessary to discuss systematically the means by which we might hope to retard the development of these formidable evils : in some instances, these will sufficiently suggest themselves, on a rational consideration of the subject in its pathological and therapeutic bearings; in others, science and art fail to help us. But I will conclude this paper by the mention 
of a few expedients which have sometimes succeeded in rescuing patients, for a time, from the fearful emergencies into which Organic Disease of the Heart, with secondary complications, tends to plunge them.

In case of structural Disease of the Heart, complicated with affection of the liver, in addition to the treatment for the former, it is necessary to use mercury more freely, with a view to its cholagogue effect. The combination of blue pill, squill, and digitalis, is commonly suitable; and may be given from once to three times a day, until the gums are slightly affected, or a slight bilious diarrhœe or copious diuresis ensues. In most instances, the addition of a little narcotic, such as. extract of conium, henbane, or poppy, is useful, to prevent the irritation of the medicine on the bowels. Cupping, blisters, and other counter-irritants to the right hypochondriac region, often do good for a time; after their use, and that of mercury, nitro-muriatic acid lotions and foot-baths, and the internal exhibition of extract of dandelion, with muriate of ammonia and nitrate of potass, will sometimes in a decided manner promote the secretion and ameliorate the condition of the liver. Saline purgatives, which derive freely from the portal system, are also occasionally useful, especially if there be any tendency to hæmorrhoids or ascites.

The complication of kidney-disease, evinced by the presence of albumen in the urine, is, in its early stage, by no means so intractable as is commonly supposed. Cupping and large blisters to the loins, with a few doses of hydragogue purgatives, have often succeeded in restoring the urine to a healthy state, and removing all symptoms of this complication, even where it had proceeded to the length of causing dropsy. The hydragogue most suitable to these cases, is the bitartrate of potass, in doses of from half an ounce to an ounce taken fasting, with a little ginger syrup in water. Its operation, if tardy, may be quickened by the addition of a few grains of gamboge or jalap, or a drop of croton oil ; but these offend the stomach and injure the appetite more than the cream of tartar alone. The same objection exists against elaterium, which has the further disadvantage of sometimes seriously depress. ing the heart's action; but when other means fail, we are constrained to resort to it; and althongh it gives him a terrible shake, it will sometimes rescue a patient from the rising waters of the dropsical flood. It has appeared to me to disorder least, when given in the dose of from a quarter to half a grain in a pill, with two or three grains of calomel and a drop of creasote. Mercury is generally considered unsuited to cases of albuminuria, on account of its tendency to cause excessive and depressing salivation, even in small doses : but this effect is, I believe, confined to the more advanced stages of kidney disease, in which the urine is of low specific gravity as well as albuminous. Where; together with albumen, there is a considerable amount of urinary matter, whether dissolved as urea, or precipitated as lithate of ammonia, and especially when the urive is also acid, I find mercury to induce no such excessive influence, but to be valuable in aiding the operation of other purgatives and diuretics. After blisters and hydragogue purgatives, sometimes aided by the warm hath (which, however, I have found very uncertain), have somewhat relieved the congestion of the kidneys, they become amenable to the influence of some diuretics, particularly digitalis, tarasacum, scoparium, diosma, with iodide of potassum, acetate 
of potass, benzoate of ammonia, or some of the saline diuretics before mentioned. The tincture of cantharides is also suited for this period of the treatment; and although an uncertain diuretic, and one that should not be continued if it do not speedily operate, it sometimes restores the lost powers of secretion better than any other remedy. It may be given in doses of from ten to forty minims, combined with bicarbonate of potass, mucilage, and a little henbane or conium. The longer the renal complication lasts, the more it impairs the quality of the blood, and produces spanæmia and tendency to dropsy; and it becomes an object to counteract this by the exhibition of iron. Generally, this remedy is not well borne; soon causing a diminished flow of urine and increase of albumen : but in some instances, a few grains of citrate of iron, or a few drops of the muriated tincture, have been added to a diuretic draught with safety and advantage. In proportion as the renal disease becomes confirmed and permanent by the substitution of exudation matter for the proper secreting cells, and by the subsequent fatty degeneration of this matter, or by its off-casting and the atrophy of the organ, the case becomes less and less susceptible of relief. The blood, impoverished, yet loaded with excrementitious matter, irritates all the organs and vessels through which it passes; and not only are the cavities and cellular textures distended with dropsical effusions, but low inflammations occur there also, and harrass the patient with a variety of new sufferings, before his final deliverance. In many instances, I have ascertained the presence or urea and uric acid in the serum thus effused; and $I$ have known the impregnation so strong, that the liquid, oozing from punctures in the legs, or from an unhealed tap-wound of the abdomen, has had a strong urinous smell, and has been found actually to contain more urea than the serous fluid which has passed by the kidneys. It is not marrellous if medicine can do little in such a subverted order of function and structure; but it has appeared wonderful to me how life has been retained, and the diseased heart has gone on working, in some instances; for days, and even for weeks, in apparent defiance of all that are com. monly considered as the laws of the functions. Almost the only useful treatment in this extremity, is by stimulants : and in addition to the usual vinous and spirituous forms, which are often tried in their most attractive varieties, as they may best please the palate of the poor sufferer, I may mention the chlorate of potass, as a medicine which seems, sometimes, to have a kind of reviving effect even in such extreme states. In doses of from five to fifteen grains, with carbonate of ammonia and a little syrup and water, it may be given every hour or two : it sometimes seems to rekindle the dying embers of life so distinctly, as to make one again reflect on a parallel (which has been only revired by Liebig) between vital chemistry and the process of combustion.

Pulmonary complications with Diseased Heart, in their moderate degrees and early stages, are readily reduced by cupping and blisters, or extensive rubefacients on the dorsal surface of the chest, mercury, antimony, salines, and expectorants. Attacks of asthma, connected with Diseased Heart, commonly depend on pulmonary congestion, sometimes exciting bronchial spasm, and ending in bronchial flux, and aro relieved by blisters, or other counter-irritants, antispasmodics, and expectorants or derivants. More intense degrees of congestion pass into 
pulmonary apoplexy or hepatisation, which, if extensive, may seriously impede the passage of air and blood in the lung, and may proceed to degeneration and further disorganization. These are among the 'most formidable complications of Diseased Heart; and, unless treated before they have involved much of the lung, they are among the most rapidly fatal.

Disease of the larger arteries, when in connexion with organic lesions of the heart, requires due consideration in the treatment. At its early. period, it is usually more or less inflammatory, and may require moderate antiphlogistic treatment, by small local bleedings, mercury, and iodide of potassium, with salines. But there is an early tendency to deposits, degeneration, and partial dilatation in the coats of the arteries; and to counteract these, tonic, astringent, and generally invigorating remedies, are more suited. Combinations of iodine, with nitric acid, preparations of zinc, tannic and gallic acids, and astringent chalybeates (such as the muriated tincture, and sulphate of iron with alum), have appeared to be serviceable in some of these cases, in controlling the undue pulsation and disturbance in parts. The cod liver oil, too, has proved beneficial in a few instances; probably, by softening the solid fatty matter of degenerating deposits, and thus rendering them more amenable to the processes of absorption and healthy reparation. Where more serious disease in the arteries, such as ossification, partial obliteration, aneurism, or great dilatation, has taken place, it becomes of great moment to keep the circulation as equal as possible, but without reducing its strength or impairing the quality of the blood; and in this way may be averted the further degeneration, wasting, and even death, of textures, which are apt to ensue in parts nourished by the injured vessels.

These observations have extended much further than I originally intended, yet the subject is far from being exhausted. The length of the paper, however, warns me to conclude the subject with a few words on the general regimen suitable to cases of Organic Disease of the Heart.

Throughout this paper I have endeavoured to keep in prominence the general fact, that Organic Diseases of the Heart tend to weaken, rather than to excite the bodily powers, and that therefore they are the proper objects for a sustaining and invigorating, rather than a lowering plan of treatment. The same position holds good as a general rule with regard to diet and regimen. Although exceptional deviations must be made in case of temporary plethora, inflammation, considerable congestion, and feverish states of the system, yet the diet should generally be as nutritious as the appetite and power of digestion can comfortably bear. On this subject I will quote the following conclusion of a lecture of mine, delivered fourteen years ago.

"The diet should generally be of as nourishing a quality as will suit the digestive organs, and will not produce plethora or inflammation. It is a great mistake, to suppose that Diseases of the Heart are to be cured by starving. Except when inflammation is actually present, or in case of hypertrophy with continued increased action, or where pulmonary hæmorrhage or apoplexy is threatened, the diet shonld be one of moderation, but not abstemiousness. In most cases, real strength in the organs of circulation, and in the muscles generally, is defective, whilst the irritability of the nervous system is undiminished. Now, if tone vOL. II. 
and strength are to be given.to the muscular system, and tranquillity to the nervous, by any mode of diet, it is by one of a nutritious character, with a due proportion of good animal food; and whilst it may be neces. sary to limit the proportion of the meat, and vary its mixture with less nutritious articles, especially farinaceous, according to the state of the digestive organs and the wants of the system, it is particularly important that in no case should the food, whether solid or liquid, be bulky in quantity. A distended stomach is apt, both mechanically and sympathetically, to excite or disorder the heart's action; and many are the individuals who suffer from palpitation under such an influence, while, at other times, they are scarcely sensible of it. The quantity of liquid food should also be limited, for a reason that $I$ have already stated, to keep down the mass of the circulating fluid. It is very common for patients with diseased hearts to suffer severely from drinking too freely, although it be only of tea or water. The appetite for excessive quantities of drink, is more the result of habit than of real want: by exercising a little self-denial in the first instance, the feelings of thirst gradually abate; and comfort and health are alike retained by drinking sparingly.

"The expediency of avoiding active exertion is so obvious, that I need not dwell on it; but there are particular kinds of exertion, which, without appearing to be violent, greatly try the heart's powers. I would instance walking up stairs or up hill, or using even moderate exertion in a constrained posture. Against these the patient must be particularly cautioned. I have known great improvement ensue on a patient removing from a house and country in which he encountered stairs or hills at every turn, to those in which all was nearly on a level. This point is the more important, because moderate exercise is in most cases lighly beneficial; and the more of this that can be taken in open, healthy air, without inducing fatigue, the better for the strength and regularity of all the functions. This must be done in some cases only on foot; but it is desirable to vary the mode of exercise, and to those accustomed to ride, no motion is more suitable or salutary than the gentle paces of an easy horse. Where riding is not borne, carriage exercise is the only other resource; and it may well be combined with walking, but when taken alone, it is inferior to either of the others. Travelling by easy stages about a pleasant country, is sometimes beneficial in many ways-by the amount of moderate exercise, the change of air, and the mental amusement which it affords. In the intervals of exercise, which constitute the greater part of the day, it is very essential that the body should really be at rest; and by easy horizontal or inclined postures, on sofas, couches, or other contrivances, that the labour of the heart be aided as much as possible in propelling the blood to all parts. When the eapacity for exercise fails, it is proper to assist the defective circulation, particularly in the extremities, by daily friction. In all cases this may prove salutary, by promoting the function of the extreme vessels and the skin. With the same object, it is highly important to have a sufficiency of woollen clothing; and if the skin is habitually dry, its condition may be improved by warm salt water baths. In a relaxed state of the surface, sponging freely with tepid or cold salt water or vinegar, followed by friction, proves equally shlutary. 
"Moral and mental quietude is most desirable, but here again not inactivity. According to the natural dispositions, various degrees of mental occupation and social intercourse are necessary; and so strongly is the action, and thence the structure of the heart, influenced by metaphysical causes, that there is no morbific circumstance more pernicious than violent mental passions, and no medicine, in chronic cases, so salutary as the calm enjoyment of agreeable social relations, and of amusing intellectual pursuits. We speak practically not less than postically, when we place these passions in the human heart; and as we hear of many a whole heart rent and broken with grief and trouble, or burst with unbridled passion, we may well judge what havoc these feelings may cause in a heart already unsound. It is not often ours to " minister to a mind diseased, or cleanse the stuffed bosom of that perilous stuff that weighs upon the heart;" but we may often enlist the patient's understanding and well-grounded fears on our own side, when we counsel him sobriety in his sorrow, and calmness in his care : on us may devolve the privilege and responsibility (and in no case does the conscientious physician, with his best exertions, more need Divine help to direct his judgment aright) of advising and deciding with regard to important changes in the relations and occupations of his future life."London Medical Gazette, 1838.

7, Holles Street, Cavendish Square.

\section{A CASE OF MUCO-ENTERITIS, FOLLOWED BY ACUTE PERITONITIS, TERMINATING IN EFFUSION INTO THE ABDOMINAL CAVITY, \\ RELIEVED BY PROFUSE SEROUS DISCHARGE FROY A SPONTANEOUS OPENING OF THE UMBILICUS BY ULCERATION, FOLLOWED BY PROLONGED SUPPURATION, REPEATED HEMORRHAGE, AND STERCORACEOUS VOMITING.}

By ALEXANDER KNOX, M.D., Physician to the Diepensary, Strangford, County Down, Ireland.

CAsE. J. S., an agricultural labourer, about thirty years of age, had enjoyed good health, until about a fortnight before bis application at the Strangford Dispensary. At the period alluded to, he had been confined to bed for two or three days, but the precise nature of his indisposition had not been ascertained. It had, however, caused much debility, from which he was not yet recovered. When I first saw him, Oct. 26, he complained of acute pains about the umbilicus, and costiveness; for which a draught of castor oil and laudanum was administered, and he was directed to have hot fomentations repeatedly applied to the abdomen. The bowels were moved with considerable difficulty, and the constipation was followed by obstinate facal diarrhce ; but the discharges were neither scybalous, mucous, nor błoody, nor was any tenesmus present. The patient, however, was flushed, the pulse was quick, and there was obscure abdominal tenderness, increased by pressure. Leeches and fomentations wero 